\title{
REVIEW
}

\section{The therapeutic potential of new insights into myocardial excitation-contraction coupling}

\author{
M Scoote, PA Poole-Wilson, A J Williams
}

Heart 2003;89:371-376

The physiological mechanisms that link myocyte depolarisation and contraction are referred to collectively as excitation-contraction coupling. This important process uses calcium as a second messenger to convert electrical depolarisation of the myocyte sarcolemma into the coordinated contraction of the cell's internal myofilament apparatus. The inotropic properties of the cell are determined by the efficiency of this process and when this efficiency is lost contractile dysfunction and heart failure develop, along with a propensity for arrhythmias. Previous attempts to use positive inotropic drugs in the management of chronic heart failure have been disappointing. Such drugs have been associated with unacceptable side effects and worse morbidity and mortality outcomes, primarily through their non-specific amplification of intracellular cascade pathways that modify the cell's inotropic state. As a result of recent advances in our understanding of how excitation-contraction coupling works in both health and disease it may be possible to design more specifically targeted drug treatment that has the potential to avoid the detrimental effect of currently available drugs while at the same time improving the inotropic properties of the cell.

See end of article for authors' affiliations

Correspondence to: Dr Mark Scoote, Department of Cardiac Medicine, National Heart and Lung Institute, Imperial College of Science, Technology and Medicine, Dovehouse Street, London SW3 6LY, UK;

mark.scoote@ic.ac.uk

Accepted

21 November 2002

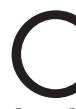
ardiac excitation-contraction (E-C) coupling refers to the process whereby myo2 cyte excitation (in the form of membrane depolarisation) is used as the stimulus for a coordinated movement of calcium around the cell to bring about contraction. ${ }^{1}$ It is a highly evolved and efficient amplification system, which uses both intracellular and sarcolemmal bound components. Great advances have been made over recent years in our understanding of how these components work together and are regulated under normal physiological conditions. Cardiac E-C coupling plays a vital role in maintaining the normal inotropic and electrical properties of the heart and it is not surprising that when this process is defective both heart failure and cardiac arrhythmias can result. Previous inotropic treatments that non-specifically amplified cardiac E-C coupling gain have largely been discredited as effective treatments in chronic heart failure. ${ }^{2}$ The design of therapeutic strategies that use our increased understanding of this process to target specific defects within it represents a new oppor- tunity in drug development that will avoid many of the limitations seen with currently available drugs.

\section{E-C COUPLING IN THE NORMAL HEART}

The key cellular components of cardiac E-C coupling and calcium movement around the myocyte are outlined in fig 1 . When the myocyte depolarises, extracellular $\mathrm{Ca}^{2+}$ enters the cell through the L type voltage dependent (dihydropyridine sensitive) calcium channel, a phenomenon represented by the phase 2 plateau of the cardiac action potential. ${ }^{3}$ This inward $\mathrm{Ca}^{2+}$ current is, on its own, insufficient to bring about the required conformational change in troponin needed for contraction to occur. Additional calcium is required and this is obtained from a pool of stored calcium within the sarcoplasmic reticulum (SR) of the cell. The initial inward movement of $\mathrm{Ca}^{2+}$ acts as an amplification signal for the release of this storage pool of SR calcium. This process, known as "calcium induced calcium release", ${ }^{4}$ occurs through an SR membrane ion channel known as the cardiac ryanodine receptor (RyR2). Individual populations of RyR2 localise in areas of the SR membrane, adjacent to L type voltage dependent channels within the $\mathrm{T}$ tubules of the sarcolemma. ${ }^{6}$ Influx of calcium through this latter channel activates its associated local population of RyR2 channels, causing a synchronised release of calcium known as a $\mathrm{Ca}^{2+}$ spark. $^{7}$ The synchronised release of multiple $\mathrm{Ca}^{2+}$ sparks throughout the cell following depolarisation creates a global intracellular $\mathrm{Ca}^{2+}$ transient of sufficient magnitude to bring about contraction (fig 2 ). Conversely, myocyte relaxation results from closure of RyR2 and the rapid removal of cytosolic calcium, either by reuptake into the SR through the sarcoplasmic $\mathrm{Ca}^{2+} /$ ATPase pump (SERCA), or by its efflux out of the cell through the sarcolemmal $\mathrm{Na}^{+} / \mathrm{Ca}^{2+}$ exchanger (NCX). These two processes balance the earlier cellular influx and SR release of calcium so that there is no net gain or loss of cellular calcium with each contractionrelaxation cycle.

The above channels and pumps interact with a variety of structural and regulatory proteins, the nature and function of which are gradually emerging. Of particular importance among these are the various enzymes that mediate phosphorylation of both the channels and the pumps

Abbreviations: $\mathrm{E}-\mathrm{C}$, excitation-contraction; NCX, sarcolemmal $\mathrm{Na}^{+} / \mathrm{Ca}^{2+}$ exchanger; RyR2, cardiac ryanodine receptor; SERCA, sarcoplasmic reticulum $\mathrm{Ca}^{2+} /$ ATPase pump; SR, sarcoplasmic reticulum 
Figure 1 Cardiomyocyte excitation-contraction coupling. Major components of $\mathrm{E}-\mathrm{C}$ coupling

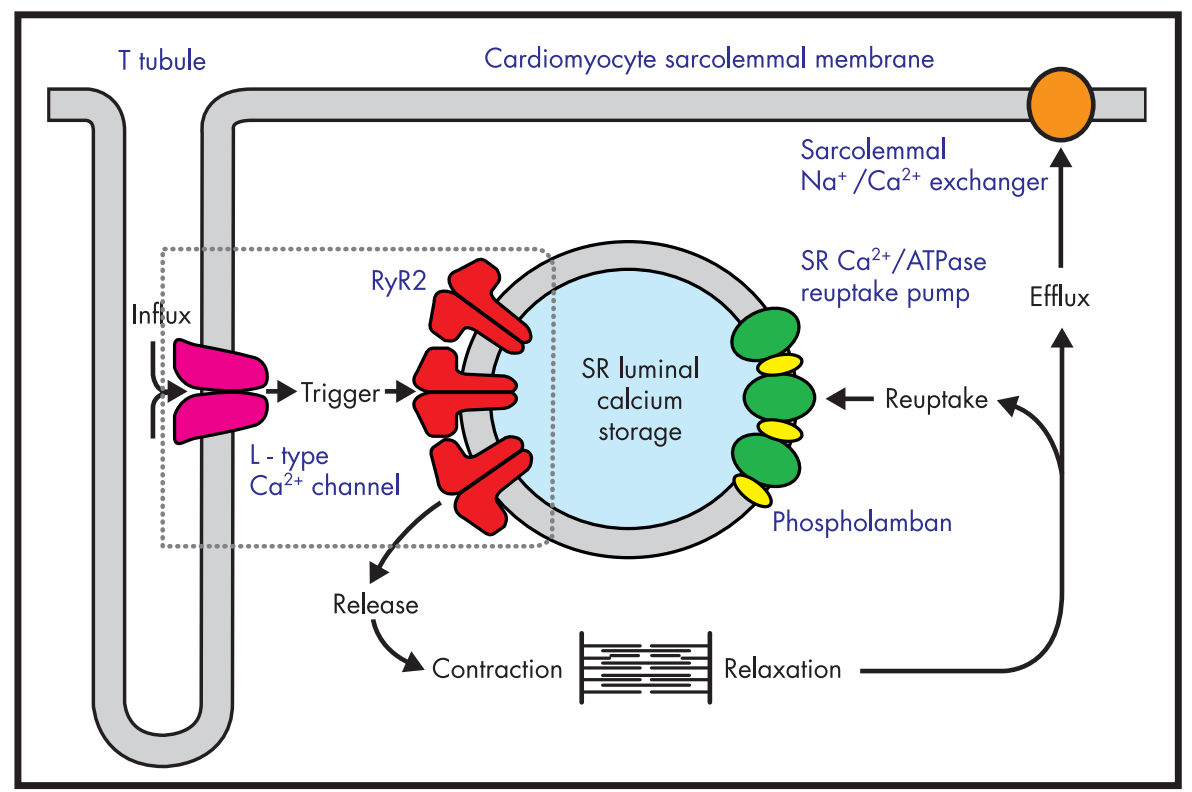
within the myocyte are shown. The movement of $\mathrm{Ca}^{2+}$ around the cell is indicated by bold arrows. Note the close association of sarcoplasmic reticulum RyR2 with the sarcolemmal L type voltage dependent channel (box), allowing localised calcium induces calcium release events to take place. SR, sarcoplasmic reticulum.

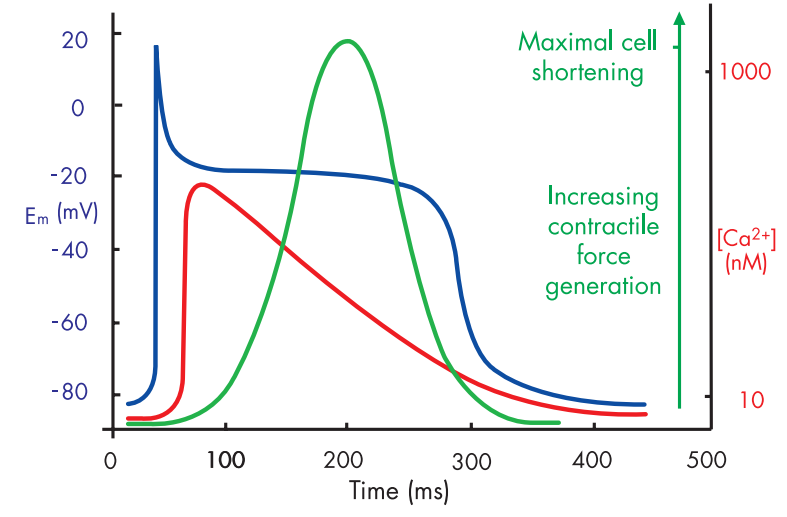

Figure 2 Diagram showing the temporal relation between the cardiac action potential (blue), the intracellular $\mathrm{Ca}^{2+}$ transient (red), and contractile force generation (green). The latter is derived from the fractional shortening of the myocyte cell length during the contraction-relaxation cycle.

themselves, and other regulatory elements attached to them. Such phosphorylation is the final event in a signal amplification cascade that begins with $\beta_{1}$ adrenergic receptor activation, either by sympathetic nerves or by circulating catecholamines. This subsequently allows the activation of adenylate cyclase and the generation of cyclic AMP, which in turn switches on phosphorylating enzymes such as protein kinase A. In this way the activity of cardiac E-C coupling can be modified by adrenergic input and this process underlies the improvements in haemodynamic variables seen with many positive inotropic drugs and the fight and flight response of the heart. Important functional consequences of phosphorylation include a greater influx of calcium through the L type voltage dependent channel for any given depolarisation and a greater release of SR calcium through RyR2. ${ }^{1}$ These directly inotropic enhancing consequences are balanced by the lusitropic effects of greater SR calcium reuptake through SERCA (as a result of phosphorylation of its regulatory protein phospholamban) and the dissociation of calcium from troponin, which occurs when this myofilament protein is phosphorylated. Although these lusitropic actions enhance myocyte relaxation they are nevertheless key to ensuring sufficient SR calcium is available for the next wave of depolarisation and thus also contribute to the overall gain in cardiac E-C coupling that adrenergic stimulation mediates.

\section{CARDIAC EXCITATION-CONTRACTION COUPLING IN THE FAILING HEART}

At the cellular level various factors may contribute towards defective contractile function in heart failure, including apoptosis, energy starvation, altered contractile protein expression, and cytoskeleton/microtubule derangements. ${ }^{8}$ In addition, defective myocyte handling of calcium also appears to be a central cause of both contractile dysfunction and arrhythmia development in heart failure. Several specific defects within the E-C coupling cascade have been detected (fig 3), but perhaps the most important of these is the observation that SR calcium stores are significantly reduced in heart failure. ${ }^{9}$ The intracellular $\mathrm{Ca}^{2+}$ transient required to initiate contraction is dependent on a sufficient SR calcium store and its subsequent release. When this store is depleted, SR calcium release will be curtailed, in terms of both its amplitude and duration, and as a result reduced contractile force is generated. ${ }^{10}$ The likely cause of this deficiency is a downregulation or reduced activity of SERCA, ${ }^{11}$ resulting in reduced loading of the SR with calcium. A further contributory factor may be upregulation of NCX, ${ }^{12}$ which also occurs in heart failure and may promote the removal of vital calcium from the cell. Reduced activity of RyR2 does not appear to be a significant factor in reduced SR calcium release; in fact there is evidence that RyR2 function may be augmented as a compensatory mechanism to overcome this deficiency. ${ }^{13}$ Over time, however, this would also serve to deplete further SR calcium stores regardless of any short term increase in calcium induced calcium release. Furthermore a diastolic leak of SR calcium (through RyR2 channels, the gating properties of which have been modified by phosphorylation) has been convincingly proposed as the mechanism whereby delayed afterdepolarisations, triggered ventricular arrhythmias, and sudden death in heart failure can occur. ${ }^{14}{ }^{15}$ In addition, upregulation of NCX has also been shown to be a proarrhythmic phenomenon which facilitates 


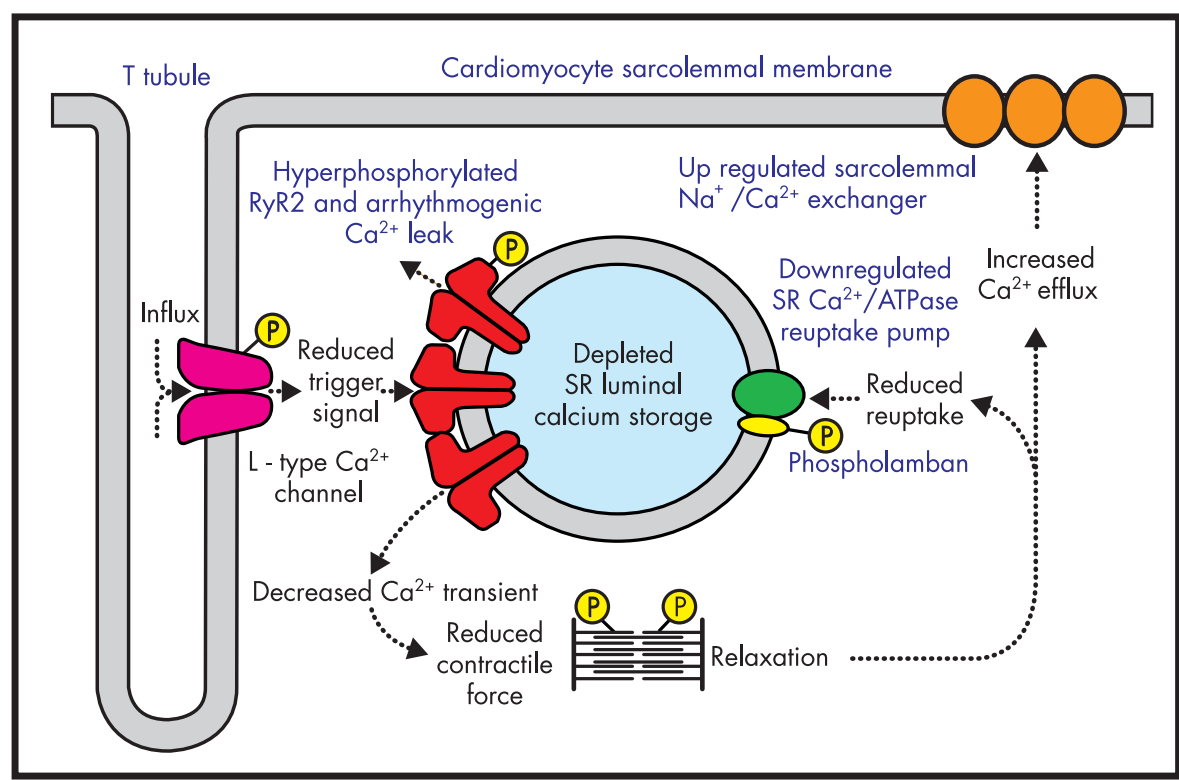

Figure 3 Deranged cardiomyocyte excitation-contraction coupling in heart failure. Intracellular catecholaminergic signal cascades upregulate the phosphorylation state of individual components in an attempt to maintain myocardial inotropy. Despite this, sarcoplasmic reticulum (SR) calcium stores are depleted and the reduced intracellular $\mathrm{Ca}^{2+}$ transient that is generated results in a loss of contractile force. An SR calcium leak through hyperphosphorylated RyR2 also contributes to proarrhythmic conditions.

the development of delayed afterdepolarisations and triggered arrhythmias in the failing heart. ${ }^{16}$

It is clear that any compensatory gain in cardiac E-C coupling mediated by adrenergic stimulation is ultimately unsuccessful in preventing the ongoing deterioration seen in untreated chronic heart failure. There is now direct evidence that in chronic heart failure, adrenergic stimulation and phosphorylation of RyR2 actually worsen cardiac function further instead of improving it. A series of experiments has shown that RyR2 becomes hyperphosphorylated in the failing heart and this leads to various functional deficiencies that increase the open probability of the channel and a tendency towards an unwanted leak of SR calcium, particularly during diastole. ${ }^{17}$ Such work is further supported by studies showing that oral $\beta$ blocker treatment reverses the above functional deficits in RyR2 and prevents hyperphosphorylation of the channel. ${ }^{18} 19$ The precise beneficial action of $\beta$ blockers in heart failure has never been adequately explained, bearing in mind their negative action on myocardial contraction in the non-failing heart. By inhibiting the excessive compensatory phosphorylation response on RyR2 in heart failure they are able to restore channel function. The gain in E-C coupling this allows is likely to contribute significantly towards their observed benefits on left ventricular function, as shown in recent trials. Furthermore by abolishing the specific diastolic leak of calcium through hyperphosphorylated RyR2 they remove the threat of arrhythmogenic delayed afterdepolarisations and are thus seen to have a valuable antiarrhythmic action in heart failure, preventing sudden arrhythmogenic death as well as improving contraction.

A further consistent experimental finding in myocytes taken from the failing heart is a functional uncoupling of the L type voltage dependent channels and their adjacent population of RyR2. ${ }^{20}$ As discussed above, the signal amplification this coupling normally allows is vital in mediating a sufficient $\mathrm{Ca}^{2+}$ transient for myocyte contraction. Without it, contraction is curtailed and this also contributes to the deteriorating inotropic state of the failing heart. One of the most likely mechanisms behind this uncoupling is a loss of the close spatial organisation between the two channels. In heart failure the extent of the sarcolemmal T tubule apparatus, which brings the L type channel into close proximity with RyR2, is reduced and this undoubtedly impairs the calcium induced calcium release amplification signal. ${ }^{21}$

\section{A NEW DIRECTION IN INOTROPIC DRUG DEVELOPMENT}

One of the main challenges facing drug developers today in the search for new compounds which influence the inotropic properties of the heart is to avoid any non-specific amplification of E-C coupling gain while at the same time addressing some of the specific functional deficits outlined above. At present circulating catecholamines and positive inotropic drugs, such as phosphodiesterase inhibitors and the various catecholamine analogues used in clinical practice, act on early steps in the signal cascade pathways that eventually mediate an increase in cardiac inotropy. At these early stages, amplified second messengers such as cyclic AMP not only act to increase cardiac inotropy but also feed into other intracellular metabolic pathways, all of which are energy consuming and increase myocardial workload. In this respect increasing cardiac inotropy through the use of such agents is highly inefficient and results in numerous unwanted intracellular consequences, which, in addition to increasing myocardial oxygen demand, are likely to contribute to the side effect profile of the agent.

What is needed in the future development of inotropic drugs are agents which specifically target the terminal effector mechanisms of the cell's inotropic state, such as the myofilament proteins themselves and the components of E-C coupling outlined above. It is much more sensible to manipulate these targets than to rely on an indirect action brought about through an action many stages higher up in the signal amplification cascade. As our understanding of how these effector mechanisms function in both health and disease has improved, new specific therapeutic targets for drug development have emerged. In some cases drugs that have taken advantage of this increased understanding have already appeared. Levosimendan, for example, is a new class of calcium sensitising drug that acts directly on the myofilament protein apparatus, sensitising it to the stimulating actions of intracellular calcium. ${ }^{22}$ This drug, which has already shown promise in clinical trials of acute heart failure management, ${ }^{23}{ }^{24}$ has emerged as a direct result of our greater understanding of the interaction between troponin and its activation by calcium. Following on from levosimendan, investigators are now looking again at the previously tarnished subject of positive inotropic drugs. Other E-C coupling components are emerging as credible specific targets for drug development, not only as a means of improving the inotropic state of the failing heart but also to address the 
problem of malignant ventricular arrhythmias in the context of heart failure.

\section{THE EMERGING ROLE OF RYR2 AS A POTENTIAL TARGET FOR ANTIARRHYTHMIC TREATMENT}

Pathological SR calcium leak, as a result of the functional deficiencies of RyR2 outlined above, appears to be an important proarrhythmic development in the failing heart, underlying delayed afterdepolarisations and the subsequent development of triggered ventricular arrhythmias. It has long been appreciated that intracellular calcium overload is a proarrhythmogenic condition and the probable cause of sudden death in patients with chronic heart failure whose mortality was increased by the use of positive inotropic drugs. The emerging causative role of RyR2 in this process offers an opportunity to address and modify such proarrhythmogenic conditions in an attempt to limit the development of fatal arrhythmias in patients with heart failure.

A greater understanding of the role played by E-C coupling components in arrhythmia generation will also come from the study of inherited arrhythmogenic disorders. Mutations in RyR2 have recently been identified as the cause of two rare inherited forms of ventricular arrhythmia and sudden cardiac death. These conditions-catecholaminergic polymorphic ventricular tachycardia ${ }^{25}$ and arrhythmogenic right ventricular dysplasia (subtype 2 ) $^{26}$-are the first examples of a specific arrhythmogenic disorder occurring secondary to defective function of one of the main E-C coupling components outlined above. Functional analysis of these RyR2 mutations is beginning to emerge ${ }^{27}$ and should help in our understanding of how specific defects in RyR2 lead to cardiac arrhythmias. Not only will this benefit sufferers from these rare disorders but it may also yield useful information about the development and mechanisms behind RyR2 induced arrhythmias in heart failure. ${ }^{15}$ Delayed afterdepolarisations have already been shown to underlie arrhythmia generation in catecholaminergic ventricular tachycardia, ${ }^{28}$ and previous studies looking at the effects of pharmacological agents that induce delayed afterdepolarisations, such as cardiac glycosides, have established that they occur secondary to an SR calcium leak. ${ }^{29}$

These pieces of evidence in combination all point to a central defect in RyR2 function as the likely cause of delayed afterdepolarisation generation and subsequent triggered ventricular arrhythmias. If this association can be confirmed in ongoing studies, a novel target for antiarrhythmic drug treatment may well be emerging. Bearing in mind that $50 \%$ or more of ventricular tachycardias in both ischaemic and non-ischaemic cardiomyopathy are thought to be initiated by non-reentrant mechanisms such as delayed after depolarisations, ${ }^{30}{ }^{31}$ the potential for an RyR2 antagonist drug that prevents proarrhythmic SR calcium leak is profound and may significantly advance the management of these arrhythmias. Although the emergence of such an agent is still some way off, drug development will be assisted by the numerous studies to date that have already used pharmacological modification of RyR2 to characterise its function and gating properties. The particular challenge for drug developers will be to produce a compound that reduces detrimental diastolic leakage of calcium through RyR2, while at the same time preserving or even amplifying the normal physiological release of calcium through RyR2 during early systole.

\section{OTHER TARGETS FOR NEW THERAPEUTIC OPTIONS}

The discovery that $\beta$ blockers restore defective RyR2 function in the failing heart through a reversal of the hyperphosphorylated receptor state seen in this condition suggests that a specific antagonist of RyR2 phosphorylation may also be of benefit in the restoration of normal cardiac function. One particular effect of RyR2 hyperphosphorylation is to cause a dissociation of FK506 binding protein 12.6 (FKBP 12.6), an associated protein thought to have a role in the regulation of RyR2 gating and in orchestrating coupled gating between adjacent receptors. ${ }^{32}$ Its in vivo role has now been clarified by the development of FKBP 12.6 knock out mice which show increased duration and amplitude of $\mathrm{Ca}^{2+}$ sparks and an increased calcium induced calcium release gain. ${ }^{33}$ It appears that FKBP 12.6 serves to prevent excessive RyR2 opening and a subsequent detrimental SR calcium leak. FKBP 12.6 levels are known to be significantly reduced in heart failure model $\mathrm{s}^{34}$ and this observation may in part explain the abnormal SR calcium leak observed in these models. ${ }^{35}$ Together, these results suggest that a decrease in FKBP 12.6 number or function is an important pathophysiological development in heart failure and arrhythmia pathogenesis. Pharmacological manipulation of this molecule, or its association with RyR2, may also be a potential avenue for the development of new drugs.

Another component of E-C coupling that has received attention for its potentially beneficial effects on the inotropic state of the heart is SERCA and its associated regulatory protein phospholamban. ${ }^{36}$ Phospholamban inhibits SERCA and thereby reduces the rate of calcium uptake into the SR. This inhibitory influence is removed when the protein is phosphorylated, and in theory restoring the function of downregulated SERCA in heart failure could be achieved by either decreasing the inhibitory influence of phospholamban or increasing the specific activity of SERCA itself. With regard to the former strategy, attempts have been made to reduce the expression levels of phospholamban through the use of specific antisense mRNA. This has the ability to reduce phospholamban expression levels by up to $70 \%^{37}$ and initial results on the subsequent gain in SERCA function that this facilitates are very encouraging. Pharmacological manipulation to overcome the negative influence of phospholamban is also possible in the laboratory, primarily through causing its dissociation from SERCA ${ }^{38}$; ; however, this approach has yet to generate a promising agent for clinical trials. With regard to increasing the specific activity of SERCA, two approaches have been used. Pharmacological stimulation of the pump itself is possible, with several compounds showing SERCA agonist activity in vitro. ${ }^{40}{ }^{41}$ Such drug development is still confined to the laboratory and no specific agent has been put forward for large scale clinical trials. More novel and promising perhaps is the use of gene transfer techniques to increase the expression levels of SERCA as a direct action against its detrimental downregulation in heart failure. Studies using adenoviral mediated gene transfer in both animal models ${ }^{42}$ and isolated myocytes from failing human hearts ${ }^{43}$ have shown that this approach is capable of restoring impaired intracellular calcium handling and normalising contractile dysfunction. Ongoing work is expected to further clarify the potential of this technique.

The prospect of potential therapeutic developments with respect to other E-C coupling components is less encouraging. Upregulation of NCX in heart failure is believed to have negative side effects such as an increased risk of arrhythmias. No credible modification has yet emerged to restore normal myocardial function without unacceptable side effects. More work is needed to establish the precise role of NCX in contractile dysfunction and arrhythmogenesis before considering it as a potential therapeutic target. ${ }^{44}$ Similarly, the L type voltage dependent channel is unlikely to offer a new potential target for therapeutic manipulation. Calcium antagonist drugs acting on the L type channel are already in widespread use and have at best a neutral effect on myocardial inotropy. From the discussions above, an amplification of this channel's function should have a positive inotropic action and would potentially help restore the defective coupling with RyR2. Any therapeutic modification which amplifies the inward 
$\mathrm{Ca}^{2+}$ transient runs the risk of intracellular calcium overload and cardiac arrhythmias. A more sensible strategy to restore the close functional link between these two channels would be to increase the sensitivity of RyR2 with respect to its activation by the depressed inward $\mathrm{Ca}^{2+}$ transient seen in heart failure.

The lack of emerging treatments which target the NCX and the L type channel highlight our ongoing need for further studies to clarify the role of these important sarcolemmal components, both under normal physiological conditions and in heart failure. As encouraging developments with regard to RyR2 and SERCA demonstrate, when a clear picture of how these proteins work emerges, subsequent opportunities for new treatment strategies soon follow. Although recent advances have been made in the treatment of heart failure and malignant ventricular arrhythmias, many patients remain symptomatic despite the use of $\beta$ blockers, angiotensin converting enzyme inhibitors, and other currently used treatments. Furthermore many patients are prevented from obtaining the potential benefits of such drugs owing to dose limiting side effects or contraindications to their use. Mechanical treatments such as left ventricular assist devices and implantable defibrillators are limited by their cost, and increasing road safety is reducing the availability of suitable hearts for heart transplant programmes. These practical points should not be forgotten, and with cardiovascular disease set to remain a major health care burden for the foreseeable future we will need new additional treatment strategies to complement those currently available. This will only emerge on the background of an increased understanding of the pathophysiological mechanisms underlying cardiac disease, and this in turn demands a greater awareness of the physiological processes that maintain normal cardiac structure and function. It remains to be seen whether any of the recent developments in our understanding of E-C coupling will eventually yield an effective pharmacological agent. What is clear from the advances and potential opportunities outlined in this article is that our negative experiences with positive inotropic drugs during the latter part of the last century should not stop us from continually searching for new and improved treatments based on the physiological processes that underlie the heart's inotropic state.

\section{ACKNOWLEDGEMENTS}

We thank the British Heart Foundation for ongoing funding of the work in our department.

\section{Authors' affiliations}

M Scoote, P A Poole-Wilson, A J Williams, Department of Cardiac Medicine, National Heart and Lung Institute, Imperial College of Science, Technology and Medicine, London, UK

\section{REFERENCES}

1 Bers D. Cardiac excitation-contraction coupling. Nature 2002;415: 198-205.

2 Cody R. Do positive inotropic agents adversely affect survival of patients with chronic congestive heart failure. J Am Coll Cardiol $1988 ; 12: 559-61$

3 Bers D. Ca ${ }^{2+}$ influx via sarcolemmal $\mathrm{Ca}^{2+}$ channels. In: Bers D. Excitation-contraction coupling and cardiac contractile force. Dordrecht: Kluwer Academic Publishers, 2001:101-32.

4 Fabiato A. Calcium induced release of calcium from the cardiac sarcoplasmic reticulum. Am J Physiol 1983;245:C1-14.

5 Coronado R, Morrissette J, Sukhareva M, et al. Structure and function of ryanodine receptors. Am J Physiol 1994;266: C 1485-504.

6 Franzini-Armstrong C, Protasi F, Ramesh V. Shape, size, and distribution of $\mathrm{Ca}^{2+}$ release units and couplons in skeletal and cardiac muscle. Biophys J 1999;77:1528-39.

7 Cheng $\mathbf{H}$, Lederer W, Cannell M. Calcium sparks. Elementary events underlying excitation-contraction coupling in heart muscle. Science 1993;262:740-4.

8 Tombe P. Altered contractile function in heart failure. Cardiovasc Res 1998;37:367-80.
9 Hobai I, O'Rourke B. Decreased sarcoplasmic reticulum calcium content is responsible for defective excitation-contraction coupling in canine heart failure. Circulation 2001;103:1577-84.

10 Lindner M, Erdmann E, Beuckelmann DJ. Calcium content of the sarcoplasmic reticulum in isolated ventricular myocytes from patients with terminal heart failure. J Mol Cell Cardiol 1998;30:743-9.

11 Hasenfuss G, Reinecke H, Studer R, et al. Relationship between myocardial function and expression of sarcoplasmic reticulum $\mathrm{Ca}^{2+}$-ATPase in failing and non failing human myocardium. Circ Res 1994:75:434-42.

12 Hasenfuss G. Alterations of calcium regulatory proteins in heart failure. Cardiovasc Res 1998;37:279-89.

13 Yamamoto T, Yano $M$, Kohno $M$, et al. Abnormal calcium release from cardiac sarcoplasmic reticulum in tachycardia induced heart failure. Cardiovasc Res 1999:44:146-55.

14 Marks A, Reiken S, Marx S. Progression of heart failure: is protein kinase a hyperphosphorylation of the ryanodine receptor a contributing factor? Circulation 2002;105:272-5.

15 Marks A, Priori S, Memmi M, et al. Involvement of the cardiac ryanodine receptor/calcium release channel in catecholaminergic polymorphic ventricular tachycardia. J Cell Physiol 2002;190:1-6.

16 Pogwizd S, Schlotthaver K, Li L, et al. Arrhythmogenesis and contractile dysfunction in heart failure: roles of sodium-calcium exchange, inward rectifier potassium current and residual $\beta$ adrenergic responsiveness. Circ Res 2001;88:1159-67.

17 Marks A. Ryanodine receptors in heart failure and sudden cardiac death. J Mol Cell Cardiol 2001;33:615-24.

18 Reiken S, Gaburjakova M, Gaburjakova J, et al. $\beta$-Adrenergic receptor blockers restore cardiac calcium release channel (ryanodine receptor) structure and function in heart failure. Circulation 2001;104:2843-8.

19 Doi M, Yano M, Kobayashi S, et al. Propranolol prevents the development of heart failure by restoring fkbp 12.6 mediated stabilisation of ryanodine receptor. Circulation 2002;105:1374-9.

20 Gomez A, Valdivia $\mathrm{H}$, Cheng $\mathrm{H}$, et al. Defective excitation-contraction coupling in experimental cardiac hypertrophy and heart failure. Science 1997; 276:800-6

$21 \mathrm{He} \mathrm{J}$, Conklin M, Foell J, et al. Reduction in density of transverse tubules and L-type calcium channels in canine tachycardia-induced heart failure. Cardiovasc Res 2001;49:298-307

22 Nawarskas J, Anderson J. Levosimendan: a unique approach to the treatment of heart failure. Heart Dis 2002;4:265-71

23 Moiseyev V, Poder P, Andrejevs N, et al. Safety and efficacy of a novel calcium sensitizer, levosimendan, in patients with left ventricular failure due to an acute myocardial infarction. a randomized, placebo-controlled, double-blind study. Eur Heart J 2002;23: 1422-32

24 Follath $\mathbf{F}$, Cleland J, Just $\mathrm{H}$, et al. Efficacy and safety of intravenous levosimendan compared with dobutamine in severe low-output heart failure (the LIDO study): a randomised double blind trial. Lancet 2002;360: 196-202.

25 Priori S, Napolitano C, Tiso N, et al. Mutations in the cardiac ryanodine receptor gene underlie catecholaminergic polymorphic ventricular tachycardia. Circulation 2000;102:49-53.

26 Tiso N, Stephan D, Nava A, et al. Identification of mutations in the cardiac ryanodine receptor gene in families affected with arrhythmogenic right ventricular cardiomyopathy type 2 (ARVD2). Hum Mol Gen 2001;10:189-94.

27 Jiang D, Xiao B, Zhang L, et al. Enhanced basal activity of a cardiac calcium release channel mutant associated with ventricular tachycardia and sudden death. Circ Res 2002;91:218-25.

28 Nakajima T, Kaneko Y, Taniguchi Y, et al. The mechanism of catecholaminergic polymorphic ventricular tachycardia may be triggered activity due to delayed afterdepolarizations. Eur Heart J 1997; 18:530-1.

29 Rosen M, Danilo P. Effects of tetrodotoxin, lidocaine, verapamil and AHR-2666 on ouabain induced delayed afterdepolarizations in canine Purkinje fibres. Circ Res 1980;46:117-24.

30 Pogwizd SM, Hoyt RH, Saffitz JE, et al. Re-entrant and focal mechanisms underlying ventricular tachycardia in the human heart. Circulation 1992;86:1872-87

31 Pogwizd SM, McKenzie JP, Cain ME. Mechanisms underlying spontaneous and induced ventricular arrhythmias in patients with idiopathic dilated cardiomyopathy. Circulation 1998;98:2404-14.

32 Marx S, Gaburjakova J, Gaburiakova M, et al. Coupled gating between cardiac calcium release channels (ryanodine receptors). Circ Res 2001;88:1151-8.

33 Xin H, Senbonmatsu T, Cheng D, et al. Oestrogen protects FKBP 12.6 null mice from cardiac hypertrophy. Nature 2002;41 6:334-7.

34 Ono K, Yano M, Ohkusa T, et al. Altered interaction of FKBP 12.6 with ryanodine receptor as a cause of abnormal calcium release in heart failure. Cardiovasc Res 2000;48:323-31.

35 Yano M, Ono K, Ohkusa T, et al. Altered stoichiometry of FKBP 12.6 versus ryanodine receptor as a cause of abnormal calcium leak through ryanodine receptor in heart failure. Circulation 2000;102:2131-36.

36 Schmidt A, Edes I, Kranias E. Phospholamban: a promising therapeutic target in heart failure. Cardiovasc Drug Ther 2001;15:387-96.

37 Eizema K, Fechner H, Bezstarosti K. Adenovirus based phospholamban antisense expression as a novel approach to improve cardiac contractile dysfunction. Circulation 2000;101:2193-9.

38 McKenna E, Smith J, Coll K. Dissociation of phospholamban and nucleotide activation of cardiac sarcoplasmic reticulum $\mathrm{Ca}^{2+}$ ATPase. Biochemistry 1999;38:2444-51. 
39 Chiesi M Schwaller R. Reversal of phospholamban induced inhibition of cardiac sarcoplasmic reticulum $\mathrm{Ca}^{2+}$ ATPase by tannin. Biochem Biophys Res Commun 1994;202:1668-73.

40 Ohizumi Y, Sasaki S, Shibusawa K, et al. Stimulation of sarcoplasmic reticulum $\mathrm{Ca}^{2+}$ ATPase by gingerol analogues. Biol Pharm Bull 1996:19:1377-9.

41 Berrebi-Bertrand I, Lahouratete P, Lahouratate V, et al. Mechanism of action of sarcoplasmic reticulum calcium-uptake activators: discrimination between sarcoplasmic reticulum $\mathrm{Ca}^{2+}$ ATPase and phospholamban interaction. Eur J Biochem 1997:247:801-9.
42 Miyamoto $M$, del Monte F, Schmidt U. Adenoviral gene transfer of SERCA2a improves left ventricular function in aortic banded rats in transition to heart failure. Proc Natl Acad Sci USA 2000;97:793-8.

43 Del Monte F, Harding S, Schmidt U. Restoration of contractile function in isolated cardiomyocytes from failing human hearts by gene transfer of SERCA2a. Circulation 1999;100:2308-11.

44 Sipido $\mathrm{K}$, Volders $\mathrm{P}$, Vos $\mathrm{M}$, et al. Altered $\mathrm{Na} / \mathrm{Ca}$ exchange activity in cardiac hypertrophy and heart failure: a new target for therapy? Cardiovasc Res 2002;53:782-805.

\section{IMAGES IN CARDIOLOGY}

\section{Prevention of embolisation during percutaneous vein graft intervention using a Filter Wire distal protection device}

$\mathrm{P}$ ercutaneous coronary intervention (PCI) in saphenous vein grafts (SVGs) carries a substantial risk of no-reflow and major adverse cardiovascular events (MACE). Distal embolisation is a key contributory factor to poor outcome. A recent randomised controlled trial demonstrated a $42 \%$ reduction in MACE with the use of a distal protection device to prevent embolisation. A 71 year old man with previous bypass grafts was admitted with an acute coronary syndrome. Coronary angiography demonstrated severe proximal stenosis in the left anterior descending (LAD) vein graft (panel A). The remaining grafts were occluded.

PCI to the LAD graft was performed with adjunctive abciximab and a distal protection device (Filter Wire EX, Boston Scientific). The device consists of a 0.014 inch angioplasty guide wire incorporating a porous filter membrane (pore size $80 \mu \mathrm{m})$ suspended from a wire loop. Radio-opaque markers (arrows) are employed to position the filter beyond the target lesion. Panel B shows the location of the Filter Wire during stent deployment. PCI was carried out by direct stenting of the culprit lesion and postdilatation with a non-compliant balloon. An excellent angiographic result was achieved (panel C). Examination of the protection device after removal (panels D and E) revealed multiple embolic particles captured within the filter, with appearances suggesting small platelet
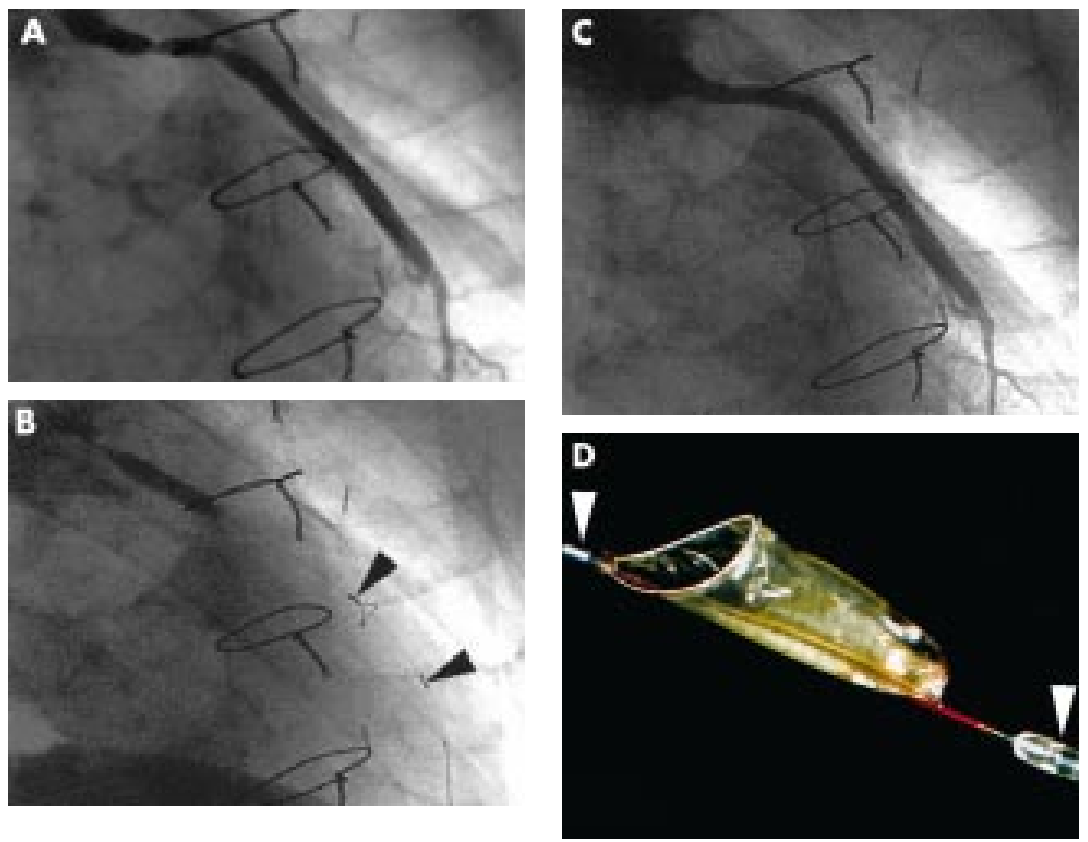

emboli (white), lipid-rich plaque debris (yellow), and acute thrombus (red). These images graphically illustrate the potential of protection devices to reduce distal embolisation during vein graft PCI.

D J Blackman

K M Channon cardiology.registrars@orh.nhs.uk

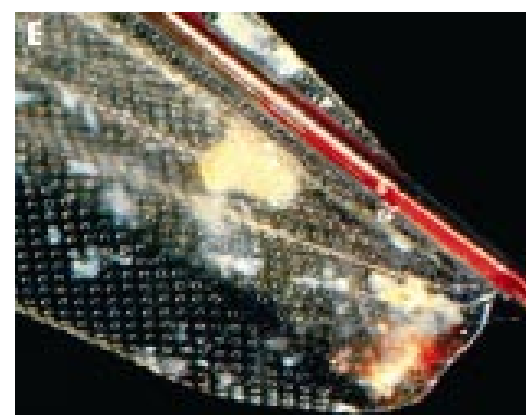

